\title{
X-rays from the High-Redshift Universe: The Chandra view
}

\author{
C. Vignali, ${ }^{1}$ W.N. Brandt, ${ }^{1}$ D.P. Schneider,${ }^{1}$ G.P. Garmire, ${ }^{1}$ S. Kaspi,${ }^{2}$ \\ F.E. BAUER, ${ }^{1}$ and D.M. ALEXANDER ${ }^{1}$ \\ 1 Dept. of Astronomy \& Astrophysics, The Pennsylvania State University, 525 Davey Lab., University Park, PA 16802, USA \\ 2 School of Physics and Astronomy, Tel-Aviv University, Tel-Aviv 69978, Israel
}

\section{Introduction}

The study of the X-ray properties of $z>4$ quasars has grown substantially over the last few years, mostly thanks to Chandra (e.g., Vignali et al. 2001, 2002; hereafter V01, V02). Here we focus on (1) the average X-ray properties of an optically selected, luminous sample of nine $z>4$ Palomar Sky Survey (PSS) quasars recently observed by Chandra, and (2) the $\mathrm{X}$-ray spectral properties of three $z>4$ AGNs in the Chandra Deep Field-North (CDF-N) using the 2 Ms exposure.

\section{The PSS sample}

This sample includes nine quasars $(z=4.09-4.51)$ observed by Chandra and selected from among the optically brightest $z>4$ PSS quasars known (V02).

- Their broad-band spectral energy distributions are characterized, on average, by steeper $\alpha_{\mathrm{ox}}$ values $\left(\left\langle\alpha_{\text {ox }}\right\rangle=-1.81 \pm 0.03\right)$ than those of lower-luminosity, lower-redshift samples of quasars (e.g., the Bright Quasar Survey quasars at $z<0.5$ have $\left.\left\langle\alpha_{\text {ox }}\right\rangle=-1.56 \pm 0.02\right)$. A likely explanation is the $\alpha_{\mathrm{ox}}-\mathrm{UV}$ luminosity anti-correlation (e.g., Vignali, Brandt, \& Schneider 2003). For comparison, a slightly flatter value is found for the less luminous $z>4$ Sloan Digital Sky Survey quasars observed by Chandra $\left(\left\langle\alpha_{\text {ox }}\right\rangle=-1.75 \pm 0.03 ; \mathrm{V} 02 ; \mathrm{V} 01\right)$.

- Using all of the Chandra $z>4$ quasars, a significant (99.99\% confidence level) correlation between $A B_{1450(1+z)}$ magnitude and soft $\mathrm{X}$-ray flux is found.

- An unabsorbed $\left(N_{\mathrm{H}} \lesssim 8.8 \times 10^{21} \mathrm{~cm}^{-2}\right.$ at the $90 \%$ confidence level) power-law model with $\Gamma=2.0 \pm 0.2$ is a reasonable fit to the joint $\approx 2-30 \mathrm{keV}$ rest-frame $\mathrm{X}$-ray spectrum of the nine PSS quasars (Fig. 1).

\section{CDF-N AGNs at $\mathrm{z}>4$}

Using the $2 \mathrm{Ms}$ exposure, we were able to perform a basic $\mathrm{X}$-ray spectral analysis for the three spectroscopically identified $z>4$ AGNs in the CDF-N at $z=5.186,4.424$, and 4.137 (Vignali et al. 2003).

- The $z=5.186$ quasar is well fitted with a power law with photon index $\Gamma=1.8 \pm 0.3$, consistent with those of lowerredshift, unobscured AGNs.

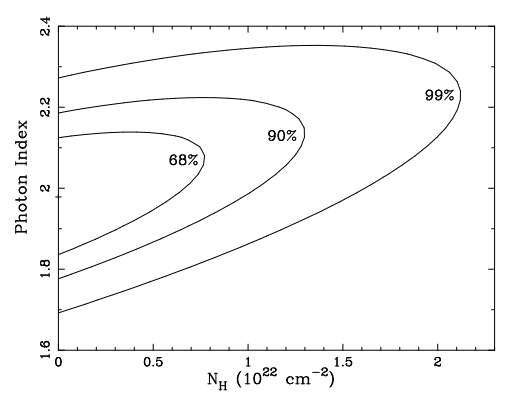

Fig. 1. Confidence regions for the photon index and intrinsic column density derived from joint spectral fitting of the nine PSS quasars.

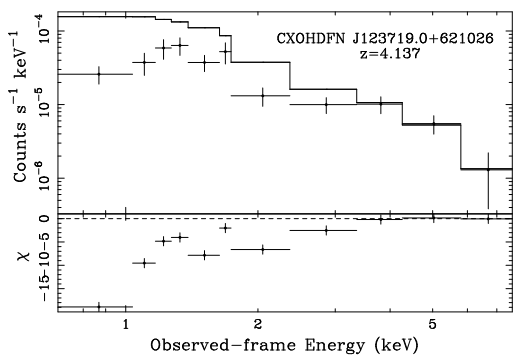

Fig. 2. The $z=4.137$ AGN X-ray spectrum fitted above $3 \mathrm{keV}$ with a $\Gamma=2$ power-law model that has been extrapolated back to lower energies. Data-to-model residuals are shown in the bottom panel (in units of $\sigma$ ). A deficit of counts below $\approx 3 \mathrm{keV}$ is present.

- The other two Seyfert-like AGNs have flatter effective $\mathrm{X}$-ray photon indices $(\Gamma \approx 1.1-1.5)$, suggesting the presence of intrinsic absorption (provided their underlying X-ray continua are similar to those of lower-redshift AGNs).

- If the $z=4.137$ AGN suffers from X-ray absorption, the implied column density is $N_{\mathrm{H}} \approx 2 \times 10^{23} \mathrm{~cm}^{-2}$ (Fig. 2).

\section{References}

Vignali, C., Brandt, W.N., Fan, X., Gunn, J.E., Kaspi, S., Schneider, D.P., Strauss, M.A.: 2001, AJ, 122, 2143 (V01)

Vignali, C., Brandt, W.N., Schneider, D.P., Garmire, G.P., Kaspi, S.: 2002, AJ, submitted (V02)

Vignali, C., Brandt, W.N., Schneider, D.P.: 2003, AJ, submitted

Vignali, C., et al.: 2003, ApJL, submitted 